\title{
CLARIFICAÇÃO DE VINHO TINTO PELO PROCESSO DE SEPARAÇÃO POR MEMBRANAS
}

\author{
B. SEGUENKA ${ }^{2}$, V. B. BRIÃO ${ }^{2}$, C. SILVEIRA ${ }^{1}$, A. C. V. SALLA ${ }^{2}$ e A. MILANI ${ }^{2}$ \\ ${ }^{1}$ Universidade Federal de Santa Catarina, Departamento de Engenharia Química e Engenharia de \\ Alimentos \\ ${ }^{2}$ Universidade Passo Fundo, Faculdade de Engenharia e Arquitetura, Curso de Engenharia de \\ Alimentos \\ E-mail para contato: brunaseguenka@hotmail.com
}

\begin{abstract}
RESUMO - A filtração por membranas tem aparecido como uma tecnologia promissora devido à sua habilidade em realizar a clarificação/filtração/higienização em uma etapa simples em operação contínua. Com o objetivo de avaliar o processo de clarificação de vinhos com o uso de membranas de ultrafiltração, realizou-se ensaios com dois tipos de membranas: uma de ultrafiltração com intervalo de separação de $80 \mathrm{KDa}$ e uma de ultrafiltração com intervalo de separação de $4 \mathrm{KDa}$ a uma pressão de 0,5 bar, temperatura de $20{ }^{\circ} \mathrm{C}$ e velocidade tangencial de alimentação de $24655 \mathrm{~m}^{3} \cdot \mathrm{h}^{-1}$. Os testes foram realizados em um equipamento piloto de laboratório e foram avaliados os seguintes parâmetros: turbidez, $\mathrm{pH}$, grau alcoólico, acidez total, extrato seco total, açucares redutores, cor, sólidos solúveis e flavonóides totais. As duas membranas de ultrafiltração testadas apresentaram reduções de turbidez, sendo a membrana de $4 \mathrm{KDa}$ mais eficiente para o processo de clarificação. Todos os vinhos apresentaram suas características físico-químicas dentro dos padrões exigidos pela legislação brasileira. Nenhuma das filtrações reteve concentrações consideráveis de flavonóides totais.
\end{abstract}

\section{INTRODUÇÃO}

O vinho é a bebida obtida da fermentação alcoólica do mosto da uva fresca, sã e madura. Do ponto de vista nutricional, os principais constituintes do vinho são: água, etanol, açúcares, minerais, vitaminas, ácidos orgânicos, aminas bioativas, e traços de proteínas (Schleier, 2004).

Os compostos fenólicos também são constituintes das uvas e por isso estão presentes tanto no vinho tinto quanto no branco. Eles desempenham uma função importante na qualidade do vinho, contribuindo para seu sabor e aroma (Pastore e Mamede, 2004).

O processo de filtração, que visa eliminar partículas em suspensão, principalmente células de fermento, deixando a bebida transparente e brilhante, dá-se o nome de clarificação (Alves et 
al., 2006). A clarificação é uma fase final do processamento dos vinho, antes do engarrafamento (Semião e Pinho, 2010).

O processo convencional de filtração usado pelas indústrias consiste de filtros de terra diatomácea os quais usam o princípio da filtração frontal (Oliveira et al., 2008). A contraindicação por esse meio de filtração são os resíduos sólidos gerados pelo uso da terra diatomácea (Carvalheira, 2010).

Os processos de separação por membranas apresentam uma série de vantagens que lhes permitem competir com as técnicas clássicas de separação. Entre estas vantagens destacam-se: economia de energia, seletividade, separação de termolábeis, simplicidade de operação e de scale up, sistemas modulares e dados para o dimensionamento facilmente obtido a partir de equipamentos pilotos operando com módulos de membrana de mesma dimensão dos utilizados industrialmente. Além disso, a operação dos equipamentos com membranas é simples e não intensiva em mão de obra (Alves et al., 2006).

A ultrafiltração (UF) é um processo intermediário referente ao tamanho dos poros, que retém tipicamente moléculas com massa molar entre 500 a 500000 Daltons (Brião e Tavares, 2005). Ela é aplicada na clarificação, na concentração de solutos e no fracionamento de componentes de uma mistura. Na indústria é usada na recuperação de tintas coloidais na pintura de veículos, na recuperação de proteínas do soro do leite, na produção de queijo, na recuperação da goma da indústria têxtil, na concentração de gelatina, na recuperação de óleos e também na clarificação do suco da maçã (Alves et al., 2006).

Alternativamente, a filtração por membranas tem aparecido como uma tecnologia promissora para realizar os processos de clarificação devido à sua habilidade em realizar a clarificação/filtração/higienização em uma etapa simples em operação contínua (Urkiaga et al., 2002).

Desse modo, o objetivo desse trabalho foi avaliar a aplicação de membranas de ultrafiltração para a clarificação de vinhos e o seu desempenho quanto à retenção/evasão das principais propriedades físico-químicas e dos flavonóides presentes no vinho.

\section{MATERIAL E MÉTODOS}

O vinho utilizado neste trabalho é proveniente da uva Vitis vinifera cultivada no interior de Passo Fundo-RS.

A clarificação do vinho foi realizada com membranas de ultrafiltrção com intervalos de separação dos poros distintos: $80 \mathrm{KDa}$ e $4 \mathrm{KDa}$.

A membrana de ultrafiltração com intervalo de separação de $80 \mathrm{KDa}$ é do tipo tubular de PVDV (policloreto de vinilideno) e possui uma área de fluxo de $0,1 \mathrm{~m}^{2}$. 
A membrana de ultrafiltração com intervalo de separação de 4 KDa é do tipo tubular de cerâmica e possui e uma área de fluxo de $0,1 \mathrm{~m}^{2}$.

As membranas utilizadas possuem uma conformação semelhante a um trocador de calor tipo casco e tubo, com 5 tubos circulares de $13 \mathrm{~mm}$ de diâmetro cada. Possuem diâmetro de 136 mm, sendo as dimensões melhor apresentadas na Figura 1.

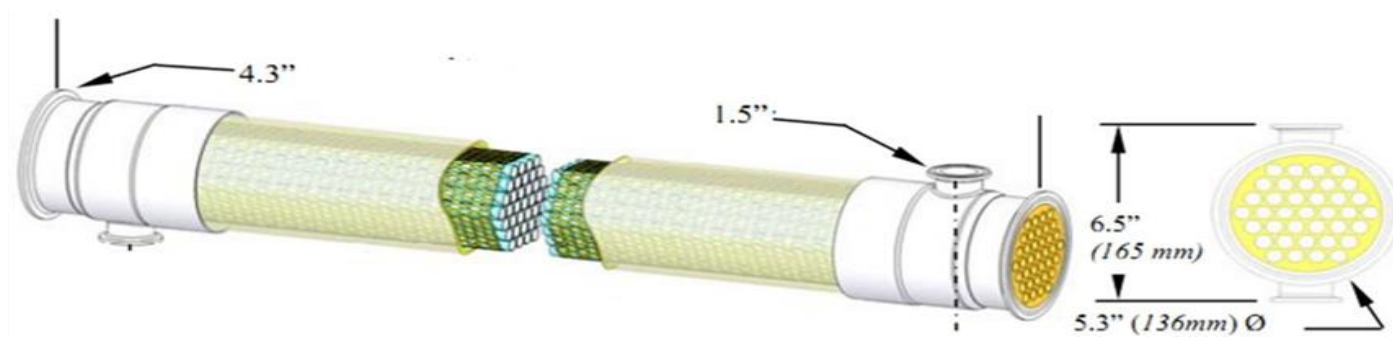

Figura 1 - Membrana piloto de filtração semelhante a um trocador de calor tipo casco e tubo. Fonte: (Koch membrane system manual, 2012)

Figura 2.

Os testes foram realizados em um equipamento piloto de laboratório, que é apresentado na

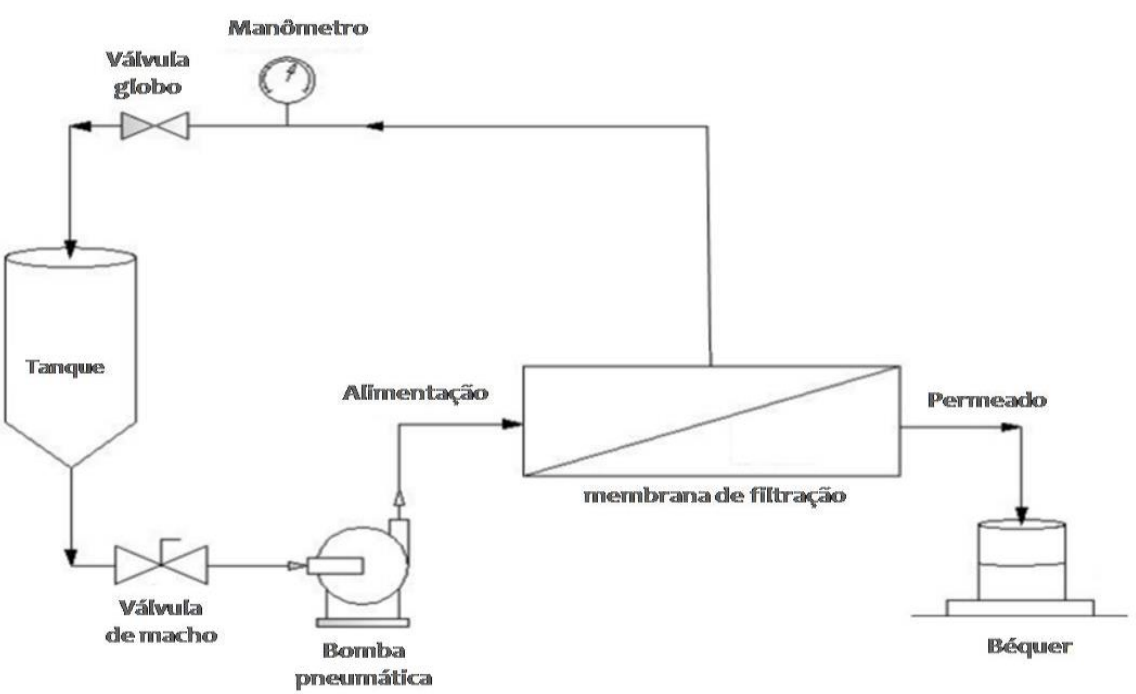

Figura 2 - Fluxograma do processo de clarificação de vinho utilizando equipamento piloto de laboratório.

Os parâmetros de filtração foram fixos para as duas membranas, sendo uma pressão de 0,5 bar, uma temperatura de $20{ }^{\circ} \mathrm{C}$ e a uma velocidade tangencial de alimentação de $24655 \mathrm{~m}^{3} \cdot \mathrm{h}^{-1}$. 
Foram avaliados para a matéria-prima e para os vinhos clarificados os seguintes parâmetros: turbidez, $\mathrm{pH}$, grau alcoólico, acidez total, extrato seco total, açucares redutores, cor, sólidos solúveis e flavonóides totais.

Para se ter um conhecimento prévio do comportamento do sistema de filtração foram feitos alguns testes preliminares para identificar as vazões de permeado em relação a diferentes pressões. Foi coletado, em uma proveta, o volume de $1 \mathrm{~L}$ de permeado, registrando o tempo em um cronômetro nas pressões de 0,5 a 3,0 bar.

A turbidez foi analisada por Turbidímetro, com realização do procedimento de acordo com Instituto Adolfo Lutz (2008). O pH foi determinado pelo método potenciométrico. O grau alcoólico foi quantificado pelo método do alcoômetro a temperatura de $20{ }^{\circ} \mathrm{C}$, de acordo com o Mapa (2010). A acidez total foi determinada pelo método titulométrico (acidimétrico) de acordo com o Mapa (2010). A determinação de extrato seco foi feita através do método de secagem em estufa a 105o de acordo com Instituto Adolfo Lutz (2008). Os açucares redutores foram quantificados pelo método de DNS de acordo com Rodrigues e Santos (2011). Os sólidos solúveis foram quantificados com a ajuda do refratômetro digital Pocket da marca ATAGO. A cor das amostras foi avaliada através do equipamento ColorQUEST II da marca HunterLab, previamente calibrado. Esse equipamento faz a leitura de cor nos parâmetros CIELab e foi usada a iluminação D65 e o observador padrão $2^{\circ}$ (Almela et al., 1995). Efetuou-se a análise do teor de compostos fenólicos totais de cada amostra espectrofotometricamente, conforme o método colorimético de Folin-Ciocalteu, utilizando ácido gálico como padrão. A metodologia para a determinação de flavonóides totais será de acordo com Sousa e Correia (2012). Para análise dos resultados foi realizada a estatística dos dados através da Análise de Variância (ANOVA) seguida do teste de Tuckey $(\mathrm{p}<0,05)$ para a comparação de médias.

\section{RESULTADOS E DISCUSSÃO}

\subsection{Caracterização da membrana de ultrafiltração de 80 kDa} Figura 3.

O teste preliminar com a membrana de ultrafiltração de $80 \mathrm{KDa}$ está representado na 


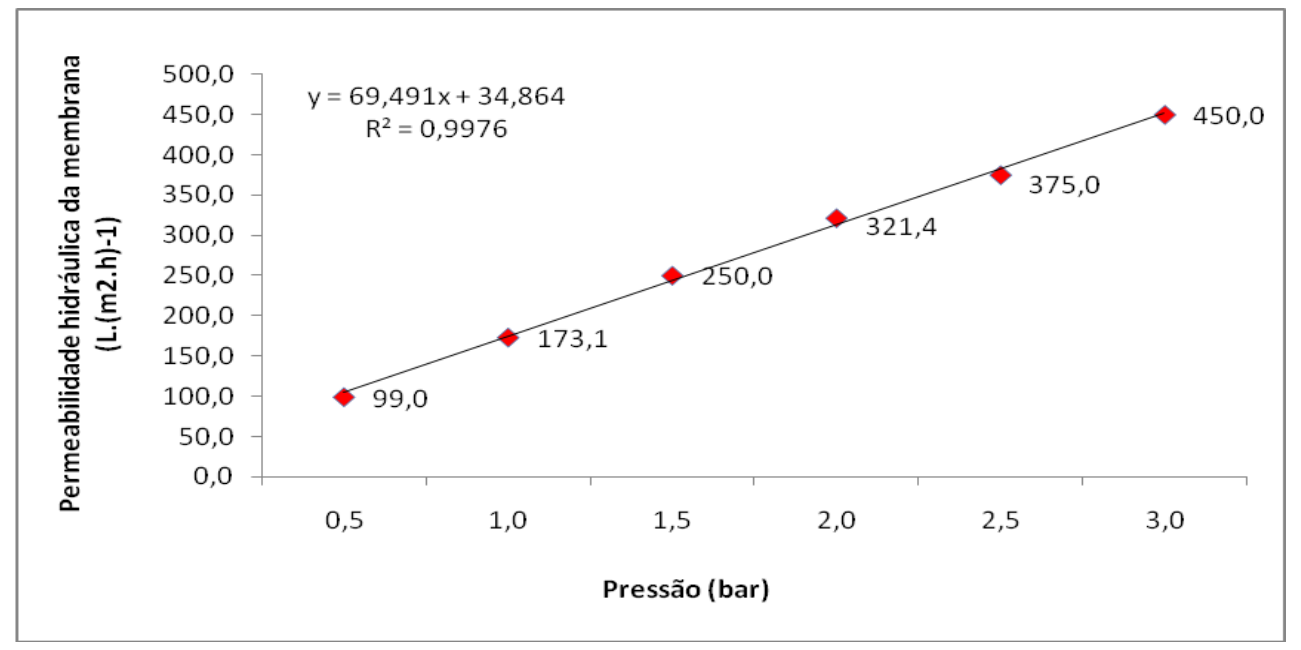

Figura 3 - Permeabilidade hidráulica da membrana de ultrafiltração com intervalo de separação de 80 KDa.

A membrana apresentou uma permeabilidade de $99 \mathrm{~L}\left(\mathrm{~m}^{2} . \mathrm{h}\right)^{-1}$, para a menor pressão avaliada (0,5 bar). Para a maior pressão $(3,0$ bar $)$, o fluxo foi de $450 \mathrm{~L}\left(\mathrm{~m}^{2} \cdot \mathrm{h}\right)^{-1}$.

\subsection{Caracterização da membrana de ultrafiltração de 4 kDa}

O teste preliminar com a membrana de ultrafiltração de 4 KDa está representado na Figura 4.

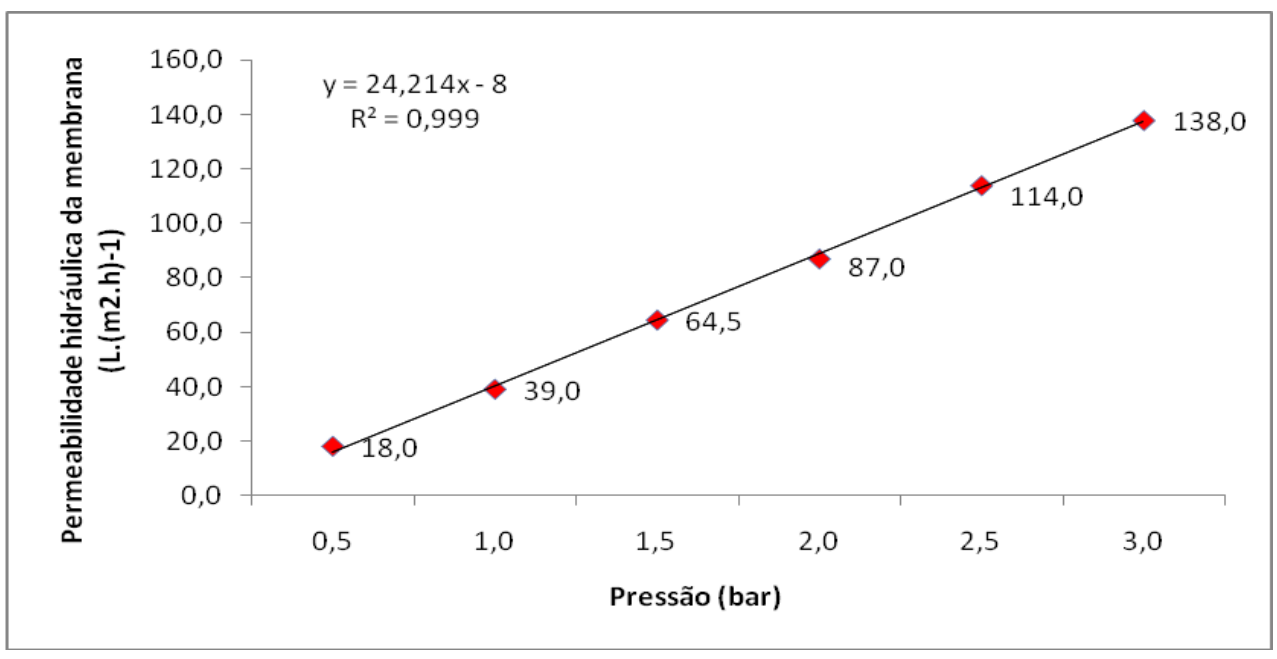

Figura 4 - Permeabilidade hidráulica da membrana de ultrafiltração com intervalo de separação de $4 \mathrm{KDa}$. 
A membrana apresentou uma permeabilidade de $18 \mathrm{~L}\left(\mathrm{~m}^{2} \cdot \mathrm{h}\right)^{-1}$, para a menor pressão avaliada (0,5 bar). Para a maior pressão $\left(3,0\right.$ bar), o fluxo foi de $138 \mathrm{~L}\left(\mathrm{~m}^{2} \cdot \mathrm{h}\right)^{-1}$.

\subsection{Caracterização físico-química da matéria-prima}

A Tabela 1 mostra os valores obtidos de turbidez, $\mathrm{pH}$, grau alcoólico, acidez total, extrato seco total, açúcares redutores, cor, sólidos solúveis e flavonóides totais para a o vinho, antes do processo de clarificação.

Tabela 1 - Valores das características físico-químicas do vinho não clarificado

\begin{tabular}{|c|c|c|c|c|c|c|c|c|c|}
\hline & $\begin{array}{c}\text { Turbidez } \\
\text { (NTU) }\end{array}$ & pH & $\begin{array}{l}\text { Álcool } \\
\left({ }^{\circ} \mathrm{GL}\right)\end{array}$ & $\begin{array}{c}\text { Acidez } \\
\text { total } \\
\left(\text { meq. } L^{-1}\right)\end{array}$ & $\begin{array}{c}\text { Extrato } \\
\text { seco } \\
\left(\text { g.L } \mathrm{L}^{-1}\right)\end{array}$ & $\begin{array}{c}\text { Açúcares } \\
\text { redutores } \\
\left(\mathbf{m g} \text { AR.L }{ }^{-1}\right)\end{array}$ & $\begin{array}{c}\text { Sólidos } \\
\text { solúveis } \\
\left({ }^{\circ} \mathrm{Brix}\right)\end{array}$ & $\begin{array}{l}\text { Cor } \\
(\Delta \mathbf{E})\end{array}$ & $\begin{array}{c}\text { Flavonóides } \\
\text { Totais } \\
\left(\mathrm{mg} \mathrm{EAG.L}^{-1}\right)\end{array}$ \\
\hline $\begin{array}{c}\text { Matéria- } \\
\text { prima }\end{array}$ & 41,2 & 3,39 & 13 & 111,01 & 17,04 & 297,80 & 5,9 & 22,20 & 1512,38 \\
\hline
\end{tabular}

O vinho que foi submetido à clarificação possuía uma turbidez de 41,2 NTU, comprovando a necessidade de clarificação.

\section{4 Ánalises físico-químicas do vinho clarificado}

Os valores de turbidez do vinho clarificado com os três tipos de membranas avaliadas e com terra diatomácea estão expressos na Tabela 2.

Tabela 2 - Valores de turbidez do vinho comparando os dois tipos de filtração

\begin{tabular}{cc}
\hline Membrana & Turbidez (NTU) \\
\hline Ultrafiltração $80 \mathrm{KDa}$ & $24,65 \pm 0,78^{\mathrm{a}}$ \\
Ultrafiltração $4 \mathrm{KDa}$ & $19,25 \pm 0,49^{\mathrm{b}}$ \\
\hline
\end{tabular}

* Resultados de média \pm desvio padrão; letras diferentes em uma mesma coluna correspondem à diferença significativa $(\mathrm{p}<0,05)$ pelo Teste de Tukey.

A análise estatística demonstra que a turbidez apresentou uma diferença significativa para todos os ensaios. Comparados com a turbidez do vinho antes da clarificação, 41,2 NTU, todos os ensaios mostraram redução da turbidez. O vinho clarificado pela membrana de ultrafiltração com intervalo de separação de $80 \mathrm{KDa}$ apresentou uma redução de 40,17 \%, já o vinho clarificado 
com a membrana de ultrafiltração com intervalo de separação de 4 KDa apresentou uma redução de $53,28 \%$.

Os resultados das análises físico-químicas do vinho clarificado com os dois tipos de membranas avaliadas estão expressos na Tabela 3.

Tabela 3 - Valores das características físico-químicas do vinho comparando os dois tipos de filtração

\begin{tabular}{|c|c|c|c|c|c|c|c|c|}
\hline Membrana & pH & $\begin{array}{l}\text { Álcool } \\
\left({ }^{\circ} \mathbf{G L}\right)\end{array}$ & $\begin{array}{c}\text { Acidez } \\
\text { total } \\
\left.\text { (meq. } \mathbf{L}^{-1}\right)\end{array}$ & $\begin{array}{c}\text { Extrato } \\
\text { seco } \\
\left(\text { g.L }{ }^{-1}\right)\end{array}$ & $\begin{array}{c}\text { Açúcares } \\
\text { redutores } \\
\left(\text { mg AR.L }{ }^{-1} \text { ) }\right.\end{array}$ & $\begin{array}{c}\text { Sólidos } \\
\text { solúveis } \\
\text { ( }{ }^{\circ} \text { Brix) }\end{array}$ & $\begin{array}{l}\text { Cor } \\
(\Delta \mathbf{E})\end{array}$ & $\begin{array}{c}\text { Flavonóides } \\
\text { Totais } \\
\left.\text { (mg EAG.L }{ }^{-1}\right)\end{array}$ \\
\hline $\begin{array}{l}\text { Ultrafiltração } \\
80 \mathrm{KDa}\end{array}$ & $3,26 \pm 0,01^{\mathrm{a}}$ & $9,50 \pm 0,71^{\mathrm{a}}$ & $95,28 \pm 1,39^{a}$ & $10,51 \pm 0,27^{\mathrm{a}}$ & $273,02 \pm 0,20^{\mathrm{a}}$ & $5,20 \pm 0,14^{\mathrm{a}}$ & $22,89 \pm 0,13^{a}$ & $1472,15 \pm 42,29^{a}$ \\
\hline $\begin{array}{c}\text { Ultrafiltração } 4 \\
\text { KDa }\end{array}$ & $3,19 \pm 0,01^{\mathrm{a}}$ & $9,00 \pm 0,00^{\mathrm{a}}$ & $90,37 \pm 1,39^{\mathrm{a}}$ & $9,91 \pm 0,07^{\mathrm{a}, \mathrm{b}}$ & $270,36 \pm 0,05^{\mathrm{a}}$ & $4,80 \pm 0,71^{\mathrm{a}}$ & $22,50 \pm 0,22^{\mathrm{a}}$ & $1395,83 \pm 39,38^{a}$ \\
\hline
\end{tabular}

* Resultados de média \pm desvio padrão; letras diferentes em uma mesma coluna correspondem à diferença significativa $(\mathrm{p}<0,05)$ pelo Teste de Tukey.

A redução de estrato seco de todos os experimentos, comparado com o valor de estrato seco do vinho antes da clarificação $\left(17,04\right.$ g. $\left.\mathrm{L}^{-1}\right)$, mostraram redução de massa.

Todas as filtrações com membranas apresentaram uma pequena retenção quanto a composição de flavonóides totais presentes no vinho. Sendo que, a ultrafiltração com intervalo de separação de $4 \mathrm{KDa}$ foi a que apresentou uma maior retenção no valor de $8 \%$. Essa pequena redução pode ter sido ocasionada pela volatilização dos polifenóis durante os processos de filtração.

De acordo com Di Stefano e Cravero (1990) o teor em flavonóides totais varia entre 930 e $2169 \mathrm{mg} . \mathrm{L}^{-1}$ e os flavonóides não antociânicos variam entre 520 e $1500 \mathrm{mg} . \mathrm{L}^{-1}$. O que comprova que os resultados encontrados para todos os experimentos estão dentro dos padrões esperados.

\section{CONCLUSÃO}

As reduções de turbidez foram gradativas de acordo com a abertura dos poros das membranas, atingindo o resultado esperado quanto ao objetivo de redução de turbidez do vinho.

A clarificação com membranas é o melhor método para clarificação de vinhos, justamente pelo fato de a filtração com terra diatomácea deixar resíduos sólidos que não podem ser reaproveitados. 
Nenhuma das filtrações reteve concentrações consideráveis de cor e flavonóides totais, o que aprova a clarificação de vinhos por membranas quanto manutenção dos compostos antioxidantes.

O vinho não clarificado e todos os vinhos submetidos à filtração apresentaram suas características físico-químicas dentro dos padrões exigidos pela legislação brasileira.

\section{REFERÊNCIAS}

ALMELA, L.; JAVOLOY, S.; FERNANDES, L. J.; LÓPES, R. J., Compararison between the tristimulus measurements Yxy and $\mathrm{L}^{*} \mathrm{a}^{*} \mathrm{~b}^{*}$ to evaluate the colour of young red wines. Food Chem., n. 53, p. 321-327, 1995.

ALVES J.A., OLIVEIRA, R.C., INNOCENTI T.D., BARROS S.T.D., MENDES, E.S., Estudo do mecanismo de fouling em vinho e cerveja. Iniciação científica CESUMAR. UEM. Maringa - PR, 2006.

BARNABÉ, D., Produção de vinho de uvas dos cultivares Niágara, Rosada e Bordô: análises físico-químicas, sensorial e recuperação de etanol a partir do bagaço. Tese de doutorado, UEP, Faculdade de Ciências agronômicas - Campus Botucatu, SP, 2006.

BRASIL. Ministério da Agricultura. Portaria no 76 de 26 de novembro de 1986. Dispõe sobre os métodos analíticos de bebidas e vinagre. Diário Oficial da República Federativa do Brasil, Brasília, 28 nov. 1986. Disponível em: http://extranet.agricultura.gov.br/sislegis. Acesso em: 25/04/2014.

BRIÃO, V.B., TAVARES C.R.G., Ultrafiltração como processo de tratamento para o reúso de efluentes de laticínios. Deartamento de Eng. Química., UEM. Maringá - PR, 2005.

CARVALHEIRA J., Filtração dos vinhos. Curso intensivo de conservação, estabilização e engarrafamento de vinhos. Lab. de Química Enológica da DRAP, 2010. Disponível em: http://www.drapc.min-agricultura.pt/base/geral/files/filtracao_vinhos.pdf $>$. Acesso em 25/04/2014.

DI STEFANO, R.; CRAVERO, M. C.; GENTILINI, N., Metodi per lo studio dei polifenoli dei vini. L'enotecnico, p. 83-89, 1989.

IAD (Instituto Adolfo Lutz), Métodos físico-químicos para análise de alimentos. Normas Analíticas do Ins. Ad. Lutz. $4^{\circ}$ ed., $1^{\circ}$ versão eletrônica, São Paulo: IMESP, 2008.

KOCH MEMBRANE SISTEM MANUAL, Microfiltration Multitube Modules for Juice

Processing. Super-Cor XL PLUS microfiltration modules. Rev. 12-2, dez, 2012.

Disponível em: <www.kochmembrane.com>. Acesso em 25/06/2014. 
MAPA, Manual e métodos de análises de bebidas e vinagres. Ministério da Agricultura, Pecuária e Abastecimento. Edição 1. Brasil, 2010.

OLIVEIRA, R.C.; MENDES, E.S.; BARROS, S.T.D., Clarificação de vinho branco com membrana de ceramica. Braz. J. Food Technol., v. 11, n. 4, p. 305-312, out./dez., 2008.

PASTORE, G. M.; MAMEDE, M. E. O., Compostos fenólicos do vinho: estrutura e ação antioxidante. B. CEPPA, Curitiba, v. 22, n. 2, p. 233-252, 2004.

RODRIGUES, D. C. G. A.; SANTOS, T. T., Manual de práticas de processos bioquímicos. Apostila das Aulas Práticas. UERJ, Faculdade de Engenharia de Produção, Departamento de Química e Ambiental, Processos Bioquímicos, 2011.

SCHLEIER, R., Constituintes fitoquímicos de Vitis vinifera L. (uva). Monografia, Instituto Brasileiro de estudos homeopáticos, Faculdade de ciências da saúde de São Paulo, São Paulo - SP, 2004.

SEMIÃO, A.; PINHO, M. N., Membrane Processes in Wine and Dairy Industries: IPPC Database, Case Studies. Instituto de Biologia Experimental e Tecnologia, Oeiras Portugal, 2010.

SOUSA, B. A.; CORREIA, R. T. P., Phenolic content, antioxidant activity and antiamylolytic activity of extracts obtained from bioprocessed pineapple and guava wastes. Braz. J. Chem. Eng., v. 29, n. 01, p. 25 - 30, Jan./Mar., 2012.

URKIAGA, A.; DE LAS FUENTES, L.; ACILU, M.; URIARTE, J., Membrane comparison for wine clarification by microfiltration. Desalination. GAIKER Centro tecnológico Amsterdan, v. 148, n.1-3, p. 115-120, 2002.

UVIBRA, Produção de Uvas, Elaboração de Vinhos e Derivados. União Brasileira de Vitivinicultura, 1998-2004. Disponível em: < http://www.uvibra.com.br >. Acesso em: 04/04/14. 